\title{
Arsenic Resistance in Enterobacteria: its Transmission by Conjugation and by Phage
}

\author{
By H. WILLIAMS SMITH \\ Houghton Poultry Research Station, Houghton, Huntingdon, \\ Cambridgeshire, PE17 2DA
}

(Received 22 March 1978)

\begin{abstract}
A high proportion of strains of Escherichia coli (599 of 716 strains), Shigella (15 of 18), Proteus (32 of 33) and Klebsiella pneumoniae (42 of 61), but not of Salmonella (15 of 209), were resistant to sodium arsenite; the incidence of arsenite resistance was higher in animal than in human strains of $E$. coli. Transmissible arsenite resistance, which was mainly plasmid-borne, was common in resistant strains of $K$. pneumoniae (22 of 34 tested) and Salmonella (11 of 12), but not in resistant strains of E. coli (10 of 98), Proteus ( 0 of 32) or Shigella $(0$ of 6$)$. In three K. pneumoniae strains from which the arsenite resistance genes could not be transferred by direct methods, they were mobilized by implanting the conjugative plasmids F or I into them. In two $S$. typhimurium strains with transmissible arsenite resistance, the resistance genes were located in the genome of the phage with which they were lysogenized. In several of the enterobacterial strains the plasmid-borne arsenite resistance was not associated with antibiotic resistance or any other character known to be transmissible. In many of the $K$. pneumoniae strains, though, it was often associated with transmissible antibiotic, mercury and tellurite resistance and especially with transmissible lactose utilization. The available evidence strongly suggested that the arsenite resistance genes were located on the Lac plasmid. The conjugative plasmids in several of the $K$. pneumoniae strains were temperature-sensitive. Many of the arsenite-sensitive $K$. pneumoniae strains grew on culture media containing mixtures of sodium arsenite and sodium nalidixate at concentrations of each which by itself would not permit their growth. Arsenite-resistant $K$. pneumoniae cultures did not grow under such conditions but any arsenite-sensitive mutants they contained did.
\end{abstract}

\section{INTRODUCTION}

Transmissible arsenic resistance was first demonstrated by Hedges \& Baumberg (1973) in a strain of Escherichia coli in which it was associated with antibiotic resistance. Summers \& Jacoby (1977) found it in 'stock' antibiotic resistance plasmids, and Smith et al. (1978) found it in temperature-sensitive antibiotic resistance plasmids obtained from a large collection of enterobacterial strains and from sewage and polluted river water. Nakahara et al. (1977) also found a high incidence in mercury resistance-bearing isolates from hospital in-patients. Because of these findings, it was decided to survey unselected enterobacterial strains for transmissible arsenic resistance paying special attention to its relationship to other transmissible characters, especially antibiotic resistance, and to the effect of temperature on its transmission rate. The results are reported in this paper. Sodium arsenite was used throughout this study because it is much more active against enterobacteria than is sodium arsenate; the latter was only used in a confirmatory sense. Unexpectedly, all the strains of $E$. coli $\mathrm{K} 12$ maintained in this laboratory were found to be resistant to arsenite. So, as it was desirable to use this organism as a potential recipient in the transmission 
experiments, an arsenite-sensitive mutant was first obtained from one of the $\mathrm{K} 12$ strains by $N$-methyl- $N^{\prime}$-nitro- $N$-nitrosoguanidine treatment.

\section{METHODS}

Bacterial strains. All the Escherichia coli, Salmonella, Shigella, Klebsiella pneumoniae and Proteus strains surveyed for arsenite resistance were believed to be epidemiologically unrelated. They had been maintained in this laboratory for at least one year on Dorset egg medium at $5{ }^{\circ} \mathrm{C}$. A smooth plasmid-free Salmonella typhimurium strain of phage type 36 and an arsenite-sensitive mutant of a lac pro his trp phe E. coli $\mathrm{K} 12$ strain were used as potential recipients of transmissible arsenite resistance. The mutant was obtained by testing isolates of the arsenite-resistant (Asir) parent strain that had been treated with $N$-methyl- $N^{\prime}$-nitro- $N$ nitrosoguanidine according to the method of Glover (1968). It is referred to throughout as the E. coli $\mathrm{K} 12$ strain. Both potential recipient strains were spontaneous chromosomal mutants resistant to sodium nalidixate $\left(\mathrm{Nal}^{\mathrm{r}}\right)$ and rifampicin $\left(\mathrm{Rif} \mathrm{r}^{\mathrm{r}}\right)$; mutants resistant to ampicillin, streptomycin and spectinomycin were also available for transferring characters from these strains.

Cultural conditions. Unless stated otherwise, all bacterial strains were grown in $10 \mathrm{ml}$ nutrient broth or on solid media (Oxoid, no. 2) for $24 \mathrm{~h}$ at $37^{\circ} \mathrm{C}$.

Sensitivity tests with antibiotics and inorganic compounds. Antibiotics were tested by the disc diffusion method and inorganic compounds were tested by the strip diffusion method (Smith et al., 1978) using strips that had been soaked in aqueous solutions of sodium arsenite $(1 \%, \mathrm{w} / \mathrm{v})$, sodium arsenate $(10 \%, \mathrm{w} / \mathrm{v})$, mercuric chloride $(0.1 \%, \mathrm{w} / \mathrm{v})$ or potassium tellurite $(0.01 \%, \mathrm{w} / \mathrm{v})$. Minimum inhibitory concentrations were determined by the method of Smith et al. (1978).

Identification of enterobacterial strains possessing transmissible arsenite resistance. Nutrient broth was inoculated with $0.02 \mathrm{ml}$ each of broth cultures of an Asi ${ }^{\mathrm{r}}$ enterobacterial strain and either the $E$. coli $\mathrm{K} 12$ strain or the $S$. typhimurium 36 strain. After incubation, the mixed culture was left at room temperature for $24 \mathrm{~h}$ (in case the conjugative mechanism of arsenite resistance transfer was temperature-sensitive) and then $0.1 \mathrm{ml}$ was inoculated on to a plate of MacConkey agar containing sodium nalidixate $\left(20 \mu \mathrm{g} \mathrm{ml}^{-1}\right)$ and sodium arsenite $\left(40 \mu \mathrm{g} \mathrm{ml}^{-1}\right)$. Colonies that grew on this medium were picked, purified by re-plating and their identity and arsenite resistance was confirmed. When necessary, the proportion of the E. coli $\mathrm{K} 12$ or S. typhimurium 36 organisms that had acquired arsenite resistance was estimated from viable counts of mixed cultures (Miles \& Misra, 1938) on MacConkey agar containing sodium nalidixate alone or sodium nalidixate and arsenite. The above procedure was modified when Asi ${ }^{\mathrm{r}} \mathrm{K}$. pneumoniae strains were being examined by using rifampicin $\left(40 \mu \mathrm{g} \mathrm{ml}^{-1}\right)$ instead of sodium nalidixate, because sodium nalidixate-resistant mutants are common in most $K$. pneumoniae cultures (Smith, 1972).

Identification of lysogenic strains. The supernates of centrifuged 6 and $24 \mathrm{~h}$ broth cultures were spotinoculated on to plates of tryptose agar (Difco) containing $0.02 \mathrm{M}-\mathrm{CaCl}_{2}$ and sodium nalidixate $\left(20 \mu \mathrm{g} \mathrm{ml}^{-1}\right.$ ) and which had previously been inoculated with a 'lawn' of the E. coli K12 or the S. typhimurium 36 strain. After incubation, the plates were examined for phage plaques. In other phage studies in which it was essential that the supernates should contain no live bacteria, they were either filtered through a membrane filter of average pore diameter $450 \mathrm{~nm}$ or held at $58{ }^{\circ} \mathrm{C}$ for $30 \mathrm{~min}$; such preparations were always tested for bacteriological sterility.

Plasmid removal. This was attempted by serial passage of strains in broth at $44^{\circ} \mathrm{C}$ or in broth containing sodium lauryl sulphate (Tomoeda et al., 1968) or acridine orange (Hirota, 1960).

Lactose and sucrose utilization. The ability of enterobacterial strains to utilize lactose or sucrose as their sole source of energy was determined by the method of Smith et al. (1978).

\section{RESULTS}

\section{Susceptibility of enterobacterial strains to sodium arsenite}

The 1037 enterobacterial strains examined were classified according to their susceptibility to sodium arsenite in strip diffusion tests (Table 1). The markedly resistant strains grew right up to the edge of the strips; the zone of bacterial inhibition in the case of the moderately resistant strains was 3 to $7 \mathrm{~mm}$ and in the case of the sensitive strains, 11 to $20 \mathrm{~mm}$. Examination of 90 strains revealed that the minimum inhibitory concentration (m.i.c.) was 500 to $2000 \mu \mathrm{g} \mathrm{ml}^{-1}$ for the markedly resistant strains, 125 to $250 \mu \mathrm{g} \mathrm{ml}^{-1}$ for the moderately resistant strains and 8 to $32 \mu \mathrm{g} \mathrm{ml}^{-1}$ for the sensitive strains. The m.i.c. for the $S$. typhimurium 36 recipient was $16 \mu \mathrm{g} \mathrm{ml}^{-1}$ and for the Asis $E$. coli $\mathrm{k} 12$ recipient was $32 \mu \mathrm{g}$ 
Table 1. Susceptibility of enterobacterial strains to sodium arsenite: the incidence of transmissible arsenite resistance

\begin{tabular}{|c|c|c|c|c|c|c|}
\hline \multirow[b]{2}{*}{ Strains } & \multicolumn{3}{|c|}{ No. of sensitive/resistant strains } & \multirow{2}{*}{$\begin{array}{l}\text { No. examined } \\
\text { for } \\
\text { transmissible } \\
\text { resistance }\end{array}$} & \multicolumn{2}{|c|}{$\begin{array}{l}\text { No. whose resistance } \\
\text { was transmitted to: }\end{array}$} \\
\hline & Sensitive & $\begin{array}{l}\text { Moderately } \\
\text { resistant }\end{array}$ & $\begin{array}{l}\text { Markedly } \\
\text { resistant }\end{array}$ & & $\begin{array}{l}\text { E. coli } \\
\mathrm{K} 12\end{array}$ & $\begin{array}{l}\text { S. typhimurium } \\
36\end{array}$ \\
\hline Salmonella spp. & 194 & 2 & 13 & 12 & 9 & 10 \\
\hline Human invasive $E$. coli & 33 & 20 & 1 & & & \\
\hline $\begin{array}{l}\text { Human enteropathogenic } \\
\text { E. coli }\end{array}$ & 28 & 54 & 2 & 98 & 10 & 4 \\
\hline Human faecal $E$. coli & 19 & 36 & 0 & & & \\
\hline Domestic animal $E$. coli & 37 & 469 & $17)$ & & & \\
\hline K. pneumoniae & 19 & 11 & 31 & 34 & 22 & 4 \\
\hline Proteus spp. & 1 & 17 & 15 & 32 & 0 & 0 \\
\hline Shigella spp. & 3 & 15 & 0 & 6 & 0 & 0 \\
\hline
\end{tabular}

$\mathrm{ml}^{-1}$; the m.i.c. for the Asir ${ }^{\mathrm{r}}$ E. coli $\mathrm{K} 12$ strain, from which the recipient was derived, and for other $E$. coli $\mathrm{K} 12$ strains maintained in this laboratory was $250 \mu \mathrm{g} \mathrm{ml}^{-1}$.

Arsenite sensitivity was most common amongst the Salmonella strains, the degree of sensitivity tending to be higher in serotypes of groups B and D than in serotypes of groups C and E. Of the human $E$. coli strains examined, a higher proportion of sensitive strains was found amongst the invasive strains, i.e. those that had been isolated from generalized infections, than amongst the enteropathogenic strains or the strains isolated from the faeces of healthy human beings. A very much lower proportion of sensitive strains was found amongst strains isolated from domestic animals despite the fact that they had been isolated from similar situations. One reason for this was that arsenite sensitivity, to a certain extent, was serotype-dependent and arsenite-sensitive serotypes were more common amongst the human strains. For example, many of the invasive strains from human beings belonged to O-groups $\mathrm{O} 4, \mathrm{O} 6$ and $\mathrm{O} 18$ which were usually arsenite-sensitive, whereas most of the invasive strains from animals belonged to serotype $078: \mathrm{K} 80$, an arsenite-resistant serotype.

Arsenite sensitivity was fairly common amongst the Klebsiella pneumoniae strains examined but less so amongst the Proteus strains which were mainly P. mirabilis.

\section{Incidence of transmissible arsenite resistance}

The resistance in 43 of 182 Asi $^{r}$ enterobacterial strains examined was transmissible (Table 1). The highest incidence was amongst the resistant Salmonella strains, but if all the strains in each group of organisms are considered, irrespective of whether they were sensitive or resistant, then the highest incidence was amongst the $K$. pneumoniae strains. Transmissible resistance was found in only a low proportion of the resistant $E$. coli strains and not at all in the resistant Proteus and Shigella strains. The m.i.c. of sodium arsenite for the $E$. coli $\mathrm{K} 12$ and $S$. typhimurium 36 strains that had acquired arsenite resistance in these experiments was approximately the same as that for the strains that had donated it.

Of the 11 Salmonella strains whose resistance was transmissible, five were S. typhimurium, three were $S$. senftenberg and one each were $S$. thompson, S. agona and S. worthington. Of the $10 \mathrm{E}$. coli strains with transmissible resistance, three had been isolated from human beings, four from pigs, two from calves and one from a chicken.

Apart from two $S$. typhimurium strains, nos 345 and 348 , all the enterobacterial strains whose resistance was transmitted to the $S$. typhimurium 36 recipient also transmitted their resistance to the $E$. coli $\mathrm{K} 12$ recipient. The latter, in general, was the better recipient, especially from the $K$. pneumoniae donor strains. The rate of transfer of the arsenite resistance genes of the different enterobacterial strains varied greatly. When some of them were transferred between organisms of the $E$. coli $\mathrm{K} 12$ strain and between organisms of 
Table 2. Characters associated with the transfer of arsenite resistance from different enterobacterial strains

\begin{tabular}{|c|c|c|c|c|}
\hline \multirow[b]{2}{*}{$\begin{array}{l}\text { Character } \\
\text { transferred }\end{array}$} & \multirow[b]{2}{*}{$\begin{array}{l}\text { No. of strains } \\
\text { tested ... }\end{array}$} & \multicolumn{3}{|c|}{$\begin{array}{c}\text { No. of strains from which transfer of arsenite } \\
\text { resistance was associated with transfer of } \\
\text { another character }\end{array}$} \\
\hline & & $\begin{array}{c}\text { Salmonella spp. } \\
11\end{array}$ & $\begin{array}{c}\text { E. coli } \\
10\end{array}$ & $\begin{array}{c}\text { K. pneumoniae } \\
22\end{array}$ \\
\hline Arsenate resistance & & 11 & 10 & 22 \\
\hline Mercury resistance & & 1 & 2 & 12 \\
\hline Tellurite resistance & & 0 & 0 & 11 \\
\hline Antibiotic resistance & & 3 & 6 & 14 \\
\hline Lactose utilization & & 0 & 1 & 21 \\
\hline Sucrose utilization & & 1 & 0 & 0 \\
\hline Colicin production & & 0 & 2 & 0 \\
\hline
\end{tabular}

S. typhimurium 36, the proportion of recipient organisms that acquired them was as high as $10^{-1}$ or as low as $10^{-6}$ to $10^{-7}$. The transfer of those from eight of the $22 \mathrm{~K}$. pneumoniae strains and from one of the $10 \mathrm{E}$. coli strains was temperature-sensitive in that they were transferred at much lower rates at $37^{\circ} \mathrm{C}$ than at $28^{\circ} \mathrm{C}$ and $22^{\circ} \mathrm{C}$.

Using a tetracycline resistance determinant as marker, the conjugative plasmids $\mathrm{F}$ and I were implanted in three of the $K$. pneumoniae strains whose arsenite resistance had not been transmitted. The arsenite resistance genes in all three strains were mobilized by both $\mathrm{F}$ and $\mathrm{I}$, their subsequent transfer rates to the $E$. coli $\mathrm{K} 12$ recipient strain being $10^{-2}$ to $10^{-4}$. It was also possible to transmit the natural arsenite resistance from an $E$. coli K12 strain in which $\mathrm{F}$ was chromosomally located, but not from one in which it was extrachromosomally located, to the $E$. coli $\mathrm{K} 12$ recipient strain, suggesting that the location of the arsenite resistance genes in E. coli $\mathrm{K} 12$ is chromosomal.

\section{Characters transferred with arsenite resistance}

The characters that the two recipient strains acquired with arsenite resistance from the 43 enterobacterial donor strains are shown in Table 2. All the $\mathrm{Asi}^{\mathrm{r}}$ forms of the recipient strains were resistant to sodium arsenate. The m.i.c. of this compound for the E. coli $\mathrm{K} 12$ strain was $800 \mu \mathrm{g} \mathrm{ml}^{-1}$ and for the $S$. typhimurium 36 strain was $200 \mu \mathrm{g} \mathrm{ml}^{-1}$; the m.i.c. for the Asir forms of both strains was $6400 \mu \mathrm{g} \mathrm{ml}^{-1}$ or greater. Many of the Asir forms of both recipients had also acquired resistance to antibiotics, mercuric chloride and potassium tellurite and the ability to utilize lactose as energy source; this was particularly so in the case of the forms that had acquired their arsenite resistance from the $K$. pneumoniae donor strains. Three of the E. coli donors and seven of the Salmonella donors did not transfer any other identifiable character with arsenite resistance. These seven Salmonella donors included the two, 345 and 348 , that transmitted arsenite resistance to S. typhimurium 36 but not to E. coli $\mathrm{K} 12$.

The examination of multiple isolates of recipient organisms from matings with 14 of the $K$. pneumoniae strains that transferred arsenite and antibiotic resistance and lactose utilization, and from cultures of these strains subjected to procedures that promote plasmid loss, suggested that in six of them the genes for arsenite and antibiotic resistance and for lactose utilization were linked and that in another six the genes for arsenite resistance and for lactose utilization were linked; the arsenite resistance genes and lactose utilization genes also appeared to be linked in the three $K$. pneumoniae strains in which they were mobilized with $\mathrm{F}$ and $\mathrm{I}$ (not shown in Table 2). 


\section{Transfer of arsenite resistance by phage}

Of all the forms of the E. coli $\mathrm{K} 12$ and $S$. typhimurium 36 recipient strains that had acquired arsenite resistance from the enterobacterial strains, only two were, as a consequence, lysogenic for the recipient strain from which they had been derived. These two were $S$. typhimurium 36 that had acquired the arsenite resistance of S. typhimurium 345 and 348, the only two enterobacterial strains that could transmit their arsenite resistance to $S$. typhimurium 36 but not to the E. coli $\mathrm{k} 12$ strain. The matings of strains 345 and 348 with $S$. typhimurium 36 were repeated and broth cultures were prepared from $40 \mathrm{Asi}^{\mathrm{r}} S$. typhimurium 36 colonies from each mating; all 80 cultures were lysogenic for $S$. typhimurium 36. In addition, the two mating mixtures were inoculated on to plates of MacConkey medium containing only sodium nalidixate instead of this antibiotic and sodium arsenite. Of $40 S$. typhimurium 36 colonies tested from the mating with strain 345 , three were arsenite-resistant and lysogenic and 37 were arsenite-sensitive and non-lysogenic; the corresponding figures for the mating with strain 348 were one and 39. The mating mixtures were filtered through membrane filters and inoculated on to lawns of $S$. typhimurium 36 in a manner that yielded isolated plaques after incubation. The resistant growth within 16 plaques from each mating yielded Asir ${ }^{\mathrm{r}}$ cultures of $S$. typhimurium 36; bacterial growth picked from all of six nonplaque regions of each lawn failed to grow on arsenite-containing media. That contact with strains 345 and 348 was not necessary for S. typhimurium 36 to acquire arsenite resistance was demonstrated by using filtrates of cultures of those organisms in the mating mixtures instead of the cultures themselves. Transfer of arsenite resistance also occurred from cultures in which the donor organisms had first been killed by chloroform or by holding at $58{ }^{\circ} \mathrm{C}$ for $30 \mathrm{~min}$, procedures which did not kill the phage particles present. Cultures held at $62{ }^{\circ} \mathrm{C}$ (but not at $60^{\circ} \mathrm{C}$ ) for $30 \mathrm{~min}$ were devoid of infective phage and were unable to confer arsenite resistance on $S$. typhimurium 36. During these experiments with culture fluids of strains 345 and 348 that contained no living organisms, the resistant growth from plaques that these fluids produced on lawns of $S$. typhimurium 36 and bacterial growth from non-plaque regions of the same lawns were examined for $\mathrm{Asi}^{\mathrm{r}}$ organisms of S. typhimurium 36. All of 200 plaques of the strain 345 phage and all of 165 plaques of the stain 348 phage yielded these organisms, but none of 150 non-plaque regions from the lawns containing strain 345 phage plaques or of 165 non-plaque regions from the lawns containing strain 348 phage plaques did. The phages from some of the 345 and 348 plaques were propagated on $S$. typhimurium 36 and plaques were re-examined for ability to transmit arsenite resistance; all of them did so. Cross-resistance tests (Bail, 1923) indicated that the 345 and 348 phages were identical.

Strains 345 and 348 were examined for ability to transfer arsenite resistance to $13 \mathrm{Sal}$ monella strains belonging to eight serotypes. Transfer was achieved to nine of them, $S$. typhimurium (four strains), $S$. heidelberg (two strains), S. agona, S. infantis and $S$. gallinarum, the organisms that had acquired arsenite resistance also acquiring lysogenicity.

Using a tetracycline resistance determinant as marker, the conjugative plasmids $\mathrm{F}$ and I were implanted in strains 345 and 348 . All four $\mathrm{F}^{+}$or $\mathrm{I}^{+}$forms failed to transfer arsenite resistance to the $E$. coli $\mathrm{K} 12$ recipient. Neither could filtrates of cultures of these forms or of spontaneous mutants of 345 and 348 resistant to streptomycin, spectinomycin or sodium nalidixate transfer their antibiotic resistance to the $S$. typhimurium 36 recipient. Loss of arsenite resistance or of lysogenicity was not demonstrated in isolates of cultures of strains 345 or 348 that had been subjected to acridine orange or sodium lauryl sulphate treatment or to serial passage in broth at $44^{\circ} \mathrm{C}$. 


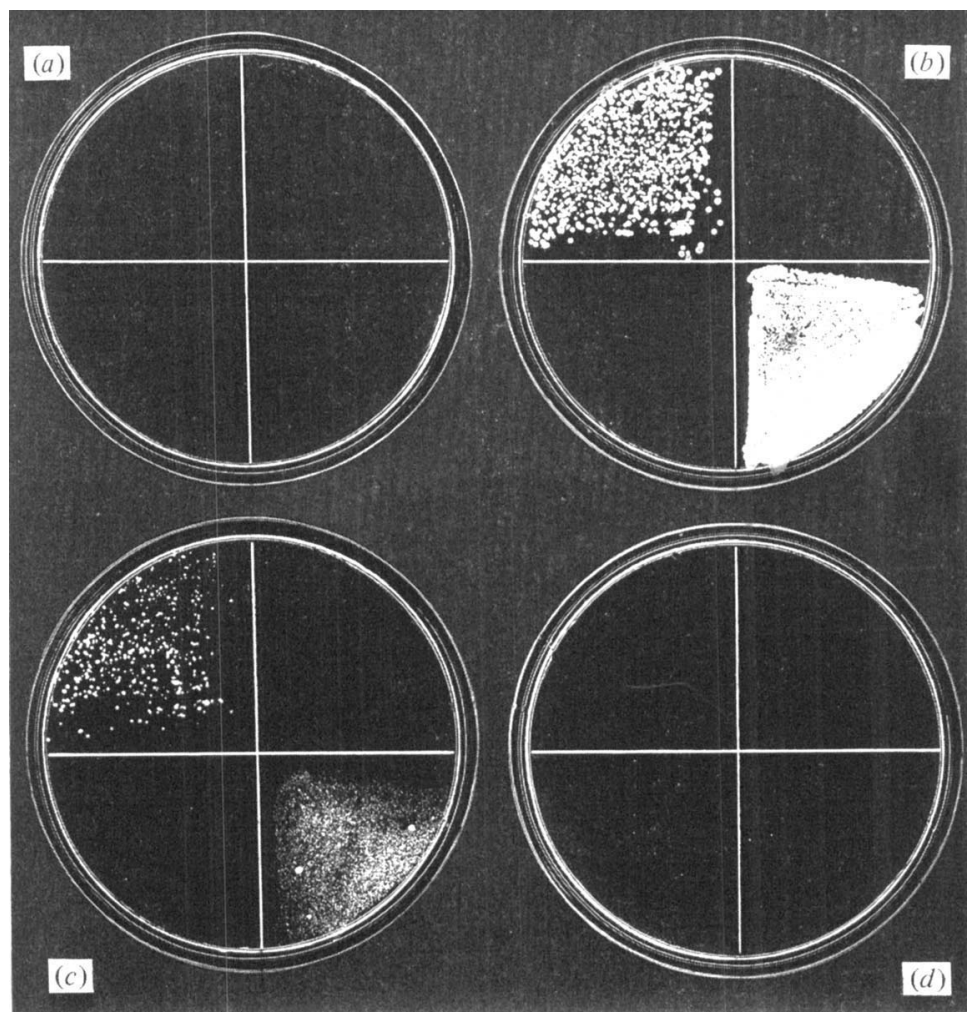

Fig. 1. Growth of $\mathrm{Asi}^{\mathrm{r}}$ and $\mathrm{Asi}^{\mathrm{s}} \mathrm{K}$. pneumoniae on Sensitest agar containing sodium nalidixate at $20 \mu \mathrm{g} \mathrm{ml}^{-1}$ and sodium arsenite at (a) $80 \mu \mathrm{g} \mathrm{ml}^{-1}$, (b) $40 \mu \mathrm{g} \mathrm{ml}^{-1},(c) 20 \mu \mathrm{g} \mathrm{ml}^{-1}$ and (d) $10 \mu \mathrm{g} \mathrm{ml}^{-1}$. The four sections of each plate were inoculated with overnight broth cultures diluted $1 / 100$ of an Asi ${ }^{\mathrm{r}}$ strain (top left) and an Asis strain (bottom right) which both gave a positive nal/asi reaction and an $\mathrm{Asi}^{\mathrm{r}}$ strain (top right) and an $\mathrm{Asi}^{\mathrm{s}}$ strain (bottom left) which did not.

\section{Effect on its virulence of implanting arsenite resistance genes in S. typhimurium 36}

Groups of five $1 \mathrm{~d}$-old chickens were each given orally approximately $10^{9}$ viable organisms of a form of the $S$. typhimurium 36 recipient strain in which had been implanted arsenite resistance genes from one of 18 of the enterobacterial donor strains. The mortality pattern in all 18 groups resembled that in uninfected control groups indicating that genes markedly affecting virulence had not been transferred with the arsenite resistance genes.

\section{Growth of Asis and Asir $K$. pneumoniae organisms in culture media containing sodium arsenite and sodium nalidixate}

Although rifampicin and sodium arsenite were used instead of sodium nalidixate and sodium arsenite for selecting organisms that had acquired arsenite resistance from the $K$. pneumoniae donor strains, because $\mathrm{Nal}^{\mathrm{r}}$ mutants are common in cultures of $K$. pneumoniae (Smith, 1972), some of the mating mixtures were inoculated on to plates of culture media containing the latter combination of antibacterial agents. On plates inoculated with some, but not all, of the mating mixtures the few donor mutant colonies that grew were greatly outnumbered by colonies of the donor strains that consisted of organisms that were $\mathrm{Nal}^{\mathrm{s}}$ and, surprisingly, Asis. Similar results were obtained when the plates were inoculated with broth cultures of the donor strains only or with cultures of some, but not all, sensitive strains of $K$. pneumoniae, the growth that occurred on plates inoculated with the latter always being heavier than that on plates inoculated with the former. This phenomenon 
which, for convenience, was referred to as the nal/asi reaction was also observed on plates of Sensitest Agar (Oxoid) but the relative proportions of sodium arsenite and sodium nalidixate in the culture medium were important for its demonstration (Fig. 1). Another method of demonstration was to lightly inoculate cultures over the surface of plates of Sensitest Agar containing sodium nalidixate $\left(20 \mu \mathrm{g} \mathrm{ml}^{-1}\right)$ and to apply a disc containing $100 \mu \mathrm{g}$ sodium arsenite to the centre of the inoculated area. After incubation, the discs on the plates that had been inoculated with $\mathrm{Asi}^{\mathrm{r}}$ and $\mathrm{Asi}^{\mathrm{s}}$ strains that gave the nal/asi reaction were surrounded by a band of bacterial growth several millimetres thick, sometimes commencing a few millimetres from the edge of the disc, and always consisting of $\mathrm{Nal}^{\mathrm{s}} \mathrm{Asi}^{\mathrm{s}}$ organisms. Similar results could be obtained by using plates containing sodium arsenite and discs containing sodium nalidixate.

Of $19 \mathrm{Asis}^{\mathrm{s}} \mathrm{K}$. pneumoniae strains tested, 15 gave the nal/asi reaction. The corresponding figure for $42^{1}$ Asi strains was 27 , strains whose arsenite resistance was known to be transmissible being more common among those that reacted positively than among those that reacted negatively. The organisms that grew from all these 27 strains of $K$. pneumoniae were Asir and some of them were also deficient in other characters possessed by their parent; six, for example, were no longer antibiotic-resistant and 17 had reduced lactose utilizing ability.

Although none of the E. coli, Salmonella, Proteus and Shigella strains had been noted to give the nal/asi reaction when they were examined for transmissible antibiotic resistance, six E. coli strains and six Salmonella strains (three of each were arsenite-resistant) were examined by the additional methods referred to above; none of the 12 gave a positive reaction.

\section{DISCUSSION}

This study has revealed the existence of transmissible arsenite resistance in Salmonella, E. coli and K. pneumoniae but not in Proteus and Shigella, the incidence of this kind of resistance, and of arsenite resistance in general, being greatly different in each of these groups of enterobacteria. Furthermore, whereas in other reports (Hedges \& Baumberg, 1973; Summers \& Jacoby, 1977; Smith et al., 1978) arsenite resistance was always associated with transmissible antibiotic resistance or transmissible mercury resistance (Nakahara et al., 1977), in the present investigation it appeared to exist in several strains in the absence of such resistance or of any other characteristic known to be transmissible. All the evidence, like that provided by the previous studies, indicated that most of the transmissible arsenite resistance was plasmid-borne. In many of the $K$. pneumoniae strains, the genes for arsenite resistance were closely associated with other plasmid-borne genes such as those for lactose utilization and for resistance to mercury, tellurite and a wide range of antibiotics. The arsenite resistance genes and the lactose utilization genes, at least, were undoubtedly located on the same plasmid and this was very probably the non-conjugative Lac plasmid of Reeve \& Braithwaite (1973). If this is always the case, then the $K$. pneumonide strains from which we transferred arsenite resistance and lactose utilization must also possess conjugative plasmids that promote their transfer. It is noteworthy that we were able to mobilize the lactose utilization and arsenite resistance genes in three $K$. pneumoniae strains by means of the conjugative plasmids $\mathrm{F}$ and $\mathrm{I}$.

The Asir $S$. typhimurium strains 345 and 348 differed from all the other strains with transmissible arsenite resistance in that their arsenite resistance genes were incorporated in the genome of a phage with which they were lysogenized. They therefore provide another naturally occurring example of resistance genes so located; the first to be reported was $\phi A \mathrm{mp}$ which contained genes for ampicillin resistance (Smith, 1972). In lysogenic organisms, $\phi$ Amp was extrachromosomally sited. We could find no evidence that the phages of strains 345 and 348 were similarly sited - they were not eliminated from organisms of these strains by 'plasmid-removing' procedures nor was the arsenite resistance of these strains trans- 
mitted to $E$. coli $\mathrm{K} 12$ after $\mathrm{F}$ and I were implanted in them. The available evidence, including that of the cross-resistance tests, indicated that the two phages were identical. However, no epidemiological relationship could be established between strains 345 and $348 ; 345$ had been isolated in 1966 and 348 in 1967.

The basis of the nal/asi reaction remains a mystery. It is not difficult to appreciate that when two compounds are added to culture media in certain proportions their antibacterial effects may be diminished. But it is not known why most Asis but not $\mathrm{Asi}^{\mathrm{r}}$ K. pneumoniae organisms grew on media containing sodium arsenite and nalidixate or why organisms of the other enterobacterial strains examined did not show this effect.

I am grateful to Miss Margaret Lovell for her capable technical help. My thanks are also due to Dr P. M. Biggs, Mr R. Sampson, Miss Susan Shaw and Professor J. T. Smith for assistance in a variety of ways.

\section{REFERENCES}

BAIL, O. (1923). Versuche über die Vielheit von Bakteriophagen. Zeitschrift für Immunitätsforschung und experimentelle Therapie 38, 57-164.

GLover, S. W. (1968). In Experiments in Microbial Genetics, p. 17. Edited by R. C. Clowes \& W. Hayes. Oxford and Edinburgh: Blackwell Scientific Publications.

Hedges, R. W. \& Baumberg, S. (1973). Resistance to arsenic compounds conferred by a plasmid transmissible between strains of Escherichia coli. Journal of Bacteriology 115, 459-460.

Hirota, Y. (1960). The effect of acridine dyes on mating type factors in Escherichia coli. Proceedings of the National Academy of Sciences of the United States of America 46, 57-64.

Miles, A. A. \& Misra, S. S. (1938). The estimation of the bactericidal power of blood. Journal of Hygiene 38, 732-749.

Nakahara, H., IshiKawa, T., Sarai, Y., Kondo, I. \& Mrtsuhashi, S. (1977). Frequency of heavy metal resistance in bacteria from inpatients in Japan. Nature, London 266, 99-101.
Reeve, E. C. R. \& Braithwaite, J. A. (1973). Lac ${ }^{+}$ plasmids are responsible for the strong lactosepositive phenotype found in many strains of Klebsiella species. Genetical Research 22, 329-333. Smith, H. Williams (1972). Ampicillin resistance in Escherichia coli by phage infection. Nature, London 238, 205-206.

Smith, H. Williams, Parsell, Z. \& Green, P. (1978). Thermosensitive antibiotic resistance plasmids in enterobacteria. Journal of General Microbiology 108, 37-47.

Summers, A. O. \& JACOBY, G. A. (1977). Plasmiddetermined resistance to tellurium compounds. Journal of Bacteriology 129, 276-281.

Tomoeda, M., INUzuKa, M., Kubo, N. \& NaKama, S. (1968). Effective elimination of drug resistance and sex factors in Escherichia coli by sodium dodecylsulphate. Journal of Bacteriology 95, 1078-1089. 